\title{
EFFECT OF THREE POLISHING SYSTEMS ON THE ROUGHNESS AND GLOSSINESS OF NANOCERAMIC COMPOSITE RESIN
}

\author{
LIDYA LOLITA, ENDANG SUPRASTIWI*, DEWA AYU NYOMAN PUTRI ARTINIGSIH, ANDRIA KUSWADI
}

Department Conservative Dentistry, Faculty of Dentistry, Universitas Indonesia, Indonesia. Email: esuprastiwi@yahoo.co.id

Received: 29 March 2020, Revised and Accepted: 9 June 2020

ABSTRACT

Objective: The purpose of resin polish is to produce a restored surface that is similar to that of enamel. The objective of this study was to analyze the polishing effect of three systems in terms of the comparative roughness or glossiness achieved with nanoceramic composite resin.

Methods: Forty samples of nanoceramic composite resin were divided into four groups as follows: Group 1 was the control group, which involved polymerization using Mylar strips; Group 2 involved a one-step polishing method; Group 3 involved a two-step method; and Group 4 involved a four-step method. After performing these methods, the comparative roughness and glossiness of the resulting surfaces were measured.

Results: Group 4 generated the lowest roughness value, with an almost equal value to that of the control group, which was followed by Group 3 and Group 2. The best surface in terms of glossiness was achieved in Group 4.

Conclusion: The polishing system involving the four-step method generated the lowest roughness value and the highest surface glossiness value.

Keywords: Nanoceramic composite resin, Polishing system, Surface roughness, Surface glossiness

(C) 2020 The Authors. Published by Innovare Academic Sciences Pvt Ltd. This is an open access article under the CC BY license (http://creativecommons. org/licenses/by/4. 0/) DOI: http://dx.doi.org/10.22159/ijap.2020.v12s2.0P-16

\section{INTRODUCTION}

In nanotechnology, nanoceramic composite resin contains methacrylatemodified polysiloxane with a glass particle size of approximately 1.1-1.5 $\mu \mathrm{m}$ and a concentration of $76 \%$ of the total weight. Nanoceramic technology offers better esthetic properties and easier handling. The nanoceramic composite resin is produced from granulation spray of ceramic particles with a filler size of less than $1 \mu \mathrm{m}$ accompanied by submicron particles and having a spherical filler shape (ball shape). The production process using spray granulation of ceramic particles $<1 \mu \mathrm{m}$ is better followed by submicron particles in the form of a spherical filler providing ease of application, fast, and easily polished.

The nanoceramic filler is a spherical mixture of pre-polymerized Sphere $\mathrm{TEC}^{\mathrm{TM}}$, which contains non-agglomerated barium glass and ytterbium fluoride [1].

The restored surface texture is important in terms of esthetics [2]. A glossier surface has better esthetic value since rougher surface facilitates both discoloration and wear [2]. In the polishing restoration process, it is important to eliminate the oxygen inhibition layer to minimize the surface roughness and ensure that a light glossiness is achieved [3]. According to Balan et al., the surface roughness value must be $<0.2 \mu \mathrm{m}[2,4]$. Van Der Vyver holds that eliminating Streptococcus bacteria are more difficult with a rough composite surface $[2,5]$.

There are many trademarked polishing systems on the market, all of which incorporate a variety of materials and polishes. However, the choice of system will determine the restorative result. The form and size of grit from the abrasive component and compact matrix flexibility as the area of abrasive planted [6,7]. The composition is classified based on the abrasive material content, which may include aluminum oxide, component carbide, abrasive diamond, oxide silicon, oxide zirconium, or silicate zirconium. Based on the abrasive composition, material polishing is divided into the areas of device and procedure. Meanwhile, the abrasive device could come in the form of a stone, disc, strip, rubber wheel, cup, or point. In terms of the procedure, this could involve a onestep method, a two-step method, or a four-step method.

There is a lack of existing research on nanoceramic composite resin materials. The current author has thus chosen to fill this gap by conducting research on nanoceramic composite resin in terms of the available polishing systems. Specifically, the main aim of the study is to analyze the effect of three polishing systems on the restored nanoceramic composite resin surfaces in terms of roughness and glossiness.

\section{METHODS}

A cylindrical mold with a diameter of $10 \mathrm{~mm}$ and a thickness of $2 \mathrm{~mm}$ was used to produce a sample of nanoceramic composite resin (ceram.X SphereTEC $^{\text {TM }}$ one universal). The composite resin sample was then divided into four groups. Group 1 was a control group that involved polymerization using Mylar strips, and then, 30 other samples were opened during the polymerization. To prevent any void in the samples, we used loads of approximately $500 \mathrm{~g}$ and polymerization using the Demi Ultra curing light (Kerr, Danbury, CT, USA). The curing light lamp had a diameter tip of approximately $11 \mathrm{~mm}$ and a curing time of approximately $10 \mathrm{~s}$. To simulate the conditions of a mouth cavity before proceeding with the polymerization, all specimens were placed in an incubator at a temperature of $37^{\circ} \mathrm{C}$ and a humidity of $100 \%$ for $24 \mathrm{~h}$. There is fixation above the sliding glass with adhesive glue for 30 specimens to the treatment group. The specimen surface is flattened with bur fine diamond to throw the rest of material redundancy around the edge of the specimen.

Meanwhile, Group 2 involved a one-step polishing method using an abrasive material containing aluminum oxide $\left(\right.$ Enhance $\left.^{\circledR}\right)$, with a rapidity of $20.000 \mathrm{rpm}$ and a one-way light pressure exerted on the conditioned dry surface for a continuous period of $20 \mathrm{~s}$. This was then followed by rinsing and drying for $6 \mathrm{~s}$, meaning the total time of the procedure was $26 \mathrm{~s}$. Group 3 involved a two-step method (EVE Diacomp Twist Plus), which began with using a pink spiral rubber wheel for the prepolishing, with a rapidity of approximately $20.000 \mathrm{rpm}$ for $20 \mathrm{~s}$ in a dry 
state before rinsing was performed with water for $6 \mathrm{~s}$. The second step involved using a gray spiral rubber wheel for the high gloss polishing, with a rapidity of approximately $20.000 \mathrm{rpm}$ for $20 \mathrm{~s}$ in a dry state before rinsing was performed with water for $6 \mathrm{~s}$. The total time for this method was $52 \mathrm{~s}$. Group 4 involved a four-step method using various disks (SofLex $^{\circledR}$ ) of coarseness. The first had a coarseness of $55 \mu \mathrm{m}$ and was applied with a rapidity of approximately $10.000 \mathrm{rpm}$ for $20 \mathrm{~s}$ before rinsing and air drying was performed for $6 \mathrm{~s}$. The second disk had a medium grit of $40 \mu \mathrm{m}$ and was applied with a rapidity of approximately $10.000 \mathrm{rpm}$ for $20 \mathrm{~s}$ before rinsing and air drying was performed for $6 \mathrm{~s}$. The third disk had a fine grit of $24 \mu \mathrm{m}$ and was applied with a rapidity of approximately $20.000 \mathrm{rpm}$ for $20 \mathrm{~s}$ before rinsing and air drying was performed for $6 \mathrm{~s}$. The last step involved applying a superfine disk $(8 \mu \mathrm{m})$ with a rapidity of approximately $20.000 \mathrm{rpm}$ for $20 \mathrm{~s}$ before rinsing and drying was performed for $6 \mathrm{~s}$. The total time of the procedure was $104 \mathrm{~s}$. The whole polishing procedure was conducted using an easy to medium constant pressure with repetitive motion to prevent warming.

Following the polishing procedures, the resulting surface roughness was analyzed using a surface roughness mean test ( $\mathrm{Ra}$, unit $\mu \mathrm{m})$. This involved the use of a surface roughness tester machine (Mitutoyo SJ301) that had been calibrated to the ISO JIS 2001 standard with a stylus rapidity distance of approximately $0.25 \mathrm{~mm} / \mathrm{s}$. Five specific surface areas for each specimen were selected for testing: Two parallel surface areas, one diagonal surface area, and one vertical surface area. The mean value was then calculated from the five resulting values.

Meanwhile, the surface glossiness was measured through a gloss meter (Novo Curve, Rhopoint, UK), which had been calibrated using a black glass standard placed on its top, which was available from the factory with a reference value of 95.5 units. The measurement was taken at the midpoint of each specimen. Four measurements were conducted with each specimen with a light incidence and reflection standpoint of $60^{\circ}$, which is vertical on toward the axis for each time specimen measurement is turn $90^{\circ}$. The mean was then calculated to obtain a single value for each specimen. The measurement area was $2 \mathrm{~mm} \times 1 \mathrm{~mm}$, while each specimen was covered by a black layer to prevent any interference from the surrounding environment. The reason for using a black layer is that this could result in reflecting inefficient light, which would affect the glossiness value.

\section{Data analysis}

The data analysis was conducted using SPSS version 21, while the data collected demonstrated a normal distribution. In addition, one-way ANOVA with a significance level of $\mathrm{p}=0.05$, which is in line with the Bonferroni post hoc test, was used to test the wealthy based on the data variants.

\section{RESULTS AND DISCUSSION}

The Shapiro-Wilks test for normality was carried out before the statistical test because the data are normally distributed, the statistical analysis is one-way ANOVA.

Table 1 shows that the lowest mean value of roughness was in Group 1 $(0.21 \mu \mathrm{m}, 0.02)$, which was followed by Group $4(0.25 \mu \mathrm{m}, 0.03)$, Group $3(0.55 \mu \mathrm{m}, 0.06)$, and Group $2(0.84 \mu \mathrm{m}, 0.09)$. Meanwhile, the highest mean value of glossiness was in Group 1 (110.27 GU, 3.51), which was followed by Group 4 (67.17 GU, 10.73), Group 3 (54.63 GU, 5.19), and Group 2 (29.81 GU, 4.81). Since the significance value was $p<0.001$, all groups showed a significant value in terms of surface roughness and surface glossiness. However, Group 4 had the smallest roughness value and the highest glossiness value.

According to the results presented in Table 2, the significance values among the groups were largely meaningless, with the exception of the roughness values for Groups 1 and 4. Therefore, the four-step method generated similar roughness results as the method used in the control group.
Table 1: Mean value, deviation standard, and significant roughness $(\mu \mathrm{m})$ and surface glossiness

\begin{tabular}{lllll}
\hline \multirow{2}{*}{ Group } & Roughness & & Glossiness & \multirow{2}{*}{$\mathbf{p}$} \\
\cline { 2 - 2 } & Mean/SD & & Mean/SD & \\
\hline 1 & $0.21 / 0.02$ & & $110.27 / 3.51$ & $0.001^{*}$ \\
2 & $0.84 / 0.09$ & & $29.81 / 4.81$ & \\
3 & $0.55 / 0.06$ & & $54.63 / 5.19$ & \\
4 & $0.25 / 0.03$ & & $67.17 / 10.73$ & \\
\hline
\end{tabular}

Group 1: Control group (a method that uses Mylar strips), Group 2=0ne-step method, Group 3=Two-step method, Group 4=Four-step method

Table 2: Significance value (p) among the groups in terms of roughness and glossiness

\begin{tabular}{lll}
\hline Group to group & Roughness & Glossiness \\
\hline $\mathbf{1}$ versus 2 & $0.001^{*}$ & $0.001^{*}$ \\
$\mathbf{1}$ versus 3 & $0.001^{*}$ & $0.001^{*}$ \\
$\mathbf{1}$ versus 4 & 1.000 & $0.001^{*}$ \\
2 versus 3 & $0.001^{*}$ & $0.001^{*}$ \\
2 versus 4 & $0.001^{*}$ & $0.001^{*}$ \\
3 versus 4 & $0.001^{*}$ & $0.001^{*}$ \\
\hline
\end{tabular}

Group 1: Control group (a method that uses Mylar strips), Group 2: One-step method, Group 3: Two-step method, Group 4: Four-step method

This study proved that there is a correlation between roughness and surface glossiness. A smoother surface has a higher glossiness value since it reflects more light, giving a glossier appearance. The high glossiness results in a more natural esthetic appearance. However, not only does a smooth surface improve the esthetic aspect, it also reduces plaque retention capacity, surface discoloration, tissue inflammation, and secondary caries and increases the patient's level of comfort. The polishing procedure is an important factor in the clinical restoration of composite resin and must be regarded as a clinical protocol.

Efforts have been made to determine which abrasive system produces the smoothest surface for composites. However, while various methods have been introduced, no single consensus has been reached in terms of which method results in the smoothest and glossiest surface.

As a control group, Group 1 only involved the use of Mylar strips to generate the smooth and glossy surface. However, here, there was a great deal of resin on the surface, which would hinder the functioning of the mouth. In addition, it made the surface comparatively rough and the restoration relatively unstable $[8,9]$. This is in line with the findings from Kormkaz et al. who stated that the composite resin surface is left covered by Mylar matrixes. The matrixes contain a great deal of resin, which hinders functioning and exposes the filler material. It is recommended not to use Mylar strips without polishing procedures [10].

The mean value of surface roughness $(\mathrm{Ra})$ should be approximately $0.2 \mu \mathrm{m}$ [3]. Our results showed that the lowest Ra value was in Group 4 (0.25), which used the four-step method, while the highest was in Group 2 (0.84), which used the one-step method. This indicates that polishing influences the restoration surface in terms of the Ra result and that the four-step system produces a smoother surface [6]. This is in line with the findings from Atabek et al., Kritzinger, Samuelson, Carvalho et al., and Goncalves and Ferracane, of whom stated that a four-step system generates smoother results with a significantly different meaning [2,3,11-15]. Indeed, several studies have shown that the more steps involved in the polishing, the better the smoothness results. This is largely due to the fact that the procedure involves a series of steps starting with coarse grits to medium, fine grains, and finally prime grits, making shallow strokes with wavelengths smaller than $0.5 \mu \mathrm{m}$. At this stroke size, the surface looks smooth and glossy $[7,12,14]$. Therefore, the current author suggests using a four-step polishing system that involves the aforementioned sequence. 
Although the roughness surface threshold is not yet agreed, this is accepted because the Ra value is more than $0.2 \mu \mathrm{m}$. It results in plaque accumulation improvement and seconder caries risk, periodontal inflammation, poor esthetics, and lower restoration endurance [7]. The restoration surface can be regarded as smooth when the roughness value is $<1 \mu \mathrm{m}$. In this case, the reflective surface was under the lighting wavelength resolution standard of less than $1 \mu \mathrm{m}[7,14,15]$. The current author suggests using a four-step polishing system to obtain the smallest roughness value and the highest glossiness value.

The particle size, the type of abrasive used in the polishing system, and the time for each procedure are all possible factors that may affect the roughness and glossiness results [11]. In this study, the four-step method used with Group 4 required the longest procedural time (approximately $104 \mathrm{~s}$ ), which was followed by that used with Group 3 (52 s) and with Group 2 (26 s), which involved the one-step polishing system. The roughness of the surface, the composite particle size, the chemical heterogeneity, and any defects and surface irregularity are among the factors that specifically affect the glossiness $[5,11]$. The characteristic of "glossy" refers to a reflection seen from a certain standpoint that involves reflected light and the level at which the light is redirected by the surface of the object. It also involves size distribution and mechanical characteristics as well as the filler refraction index and the viscosity and matrix components of the refraction index. These factors influence the glossiness in terms of the material characteristics [11]. In this study, the lowest glossiness measurement was obtained in Group 2 , while the highest was obtained in Group 4. This shows that more glossiness is generated through using more polishing steps.

Based on the ISO 2813, ASTHD 523 and 2457, and DIN 67530 standards, the surface glossiness must be measured according to an illumination angle $60^{\circ}$, which was the case with this research. This $60^{\circ}$ angle approaches the average surface angle of view normally observed by the operator [7]

The surface glossiness value tends to follow the roughness value. By a measurement angle of $60^{\circ}$, poor polishing results in values below $60 \mathrm{GU}$. The standard value falls between 60 and $70 \mathrm{GU}$, while the value of polishing using a four-step procedure falls between 70 and $80 \mathrm{GU}$. In general, the best polishing value is above $80 \mathrm{GU}$. The mean glossiness values in this study indicate that one- and two-step polishing systems produce low glossiness, while the four-step polishing system produces a standard glossiness. Meanwhile, the control group had a glossy value of over $80 \mathrm{GU}$, which is very good. Although there is a correlation between roughness and glossiness, they should be statistically analyzed separately [7].

\section{CONCLUSION}

The specific polishing system used has an influence on the roughness and glossiness of nanoceramic composite resin. The four-step polishing system generates the lowest roughness value and the highest surface glossiness value.

\section{AUTHORS' CONTRIBUTIONS}

All the authors have contributed equally.

\section{CONFLICTS OF INTEREST}

The authors declare no conflicts of interest.

\section{FUNDING}

Nil.

\section{REFERENCES}

1. Dentsply. Ceram X Universal and Duo. Vol. 27. United States: Dentsply; 2015.

2. Kritzinger D. The effect of different polishing systems on the surface roughness of a nanocomposite and a microhybrid composite. SADJ 2015;72:249-57.

3. Mopper BK. Contouring, finishing, and polishing anterior composites. Insid Dent 2011;7:62-70.

4. Balan A, Sandu A, Stoleriu S, Pintiliciuc VS, Toma V. Effect of different finishing and polishing systems on the surface roughness of composite resins. Mater Plast 2015;52:55-7.

5. Van Der Vyver P. Predictable restorations using a new nano-ceramic composite-two case studies. Int Dent 2002;6:36-46.

6. Gladwin MB. Clinical Aspects of Dental Materials. $4^{\text {th }}$ ed. Philadelphia, PA: Lippincott Williams and Wilkins; 2013.

7. Kenneth JA, Ralph WP, Chiayi S, Ralph R. Phillips' Science of Dental Materials. $12^{\text {th }}$ ed. St. Louis, Missouri: Elsevier/Saunders; 2013.

8. Watanabe T, Miyazaki M, Takamizawa T. Influence of polishing duration on surface roughness. J Oral Sci 2005;47:21-5.

9. Atabek D, Ekçi ES. The effect of various polishing systems on the surface roughness of composite resins. Acta Odontol Turc 2016;33:69-74

10. Baltacioğlu IH, Irmak O, Ulusoy N, Cengiz E. Comparison of onestep and multistep polishing systems for the surface roughness of resin composites. Open J Stomatol 2016;6:73-80.

11. Ferracane J, Paravina RD, Mazur RU. Roughness and gloss of various resin composites. J Esthet Restor Dent 2007;19:214-24.

12. Samuelson BA. Materials after finishing and polishing. Gen Dent 2015;63:26-32.

13. Carvalho F, Lins R, Ferreira RC, Pereira CN, Moreira AN, Magalhães CS. Surface roughness, microhardness, and microleakage of a silorane-based composite resin after immediate or delayed finishing/ polishing. Int J Dent 2016;2016:1-8.

14. Goncalves CF, Ferracane JL. Comparison of two-step versus four-step composite finishing/polishing disc systems: Evaluation of a new twostep composite polishing disc system. Oper Dent 2011;36:205-12.

15. Guará K, Almeida B, Guará K, Costa JF, Alves CM. Effect of different polishing systems on the surface roughness of microhybrid composites. J Appl Oral Sci 2009;17:21-6. 